\title{
Accurate Signal Detection for BPSK-OFDM Systems in Time-varying Channels
}

\author{
Min Huang ${ }^{\mathrm{a}}$, Lei Huanga,*, Weize Sun ${ }^{\mathrm{a}}$, Jihong Zhang ${ }^{\mathrm{a}}$, H. C. So ${ }^{\mathrm{b}}$ \\ ${ }^{a}$ College of Information Engineering, Shenzhen University, Shenzhen, 518060, P. R. China \\ ${ }^{b}$ Department of Electronic Engineering, City University of Hong Kong, Hong Kong
}

\begin{abstract}
Binary phase shift keying (BPSK) orthogonal frequency division multiplexing (OFDM) has been adopted in the fourth-generation wireless communications. However, the real-valued property of BPSK-OFDM signals has not yet been utilized for channel equalization. In this work, this property is employed to develop a new equalizer based on the minimum mean square error (MMSE) criterion, leading to considerable enhancement in signal detection. In particular, the proposed equalizer is able to achieve $3 \mathrm{~dB}$ improvement over the existing MMSE equalizer in large signal-to-noise ratio region, and more robust against channel estimation errors. Moreover, successive detection technique employed in the devised method is able to make use of diversity in time-varying channels, resulting in further performance enhancement. Additionally, the computational complexity of the proposed approach is analyzed. Simulation results are included to illustrate its performance superiority and computational attractiveness.
\end{abstract}

Keywords: Detection, BPSK-OFDM, MMSE, equalizer, time-varying channel 2010 MSC: 00-01, 99-00

\section{Introduction}

Increasing demands for high spectral efficiency and transmission performance have boosted the development of fourth-generation $(4 \mathrm{G})$ broadband wireless

*Corresponding author

E-mail address: lhuang@szu.edu.cn

Preprint submitted to Journal of LATEX Templates

August 12, 2016

(C) 2016. This manuscript version is made available under the Elsevier user license http://www.elsevier.com/open-access/userlicense/1.0/ 
systems. As one of the most popular modulation techniques for $4 \mathrm{G}$ systems, 5 orthogonal frequency division multiplexing (OFDM) has been adopted in many wireless communication standards, such as Wireless Fidelity (Wi-Fi), Worldwide Interoperability for Microwave Access (WiMAX), Long Term Evolution (LTE) and Digital Video Broadcasting (DVB) [1]. Usually, the OFDM system assigns symbols onto subcarriers by using discrete Fourier transform (DFT). To obtain better spectral efficiency, the OFDM scheme utilizing discrete cosine transform (DCT), denoted as DCT-OFDM, has been studied in [2-4]. In the DCT-OFDM system [2], channel equalization is carried out in frequency domain whereas signal detection is conducted in cosine domain. This, however, usually leads to noise amplification [3]. To tackle this issue, by using the relationship between the DCT and DFT, a computationally efficient equalizer has been devised for OFDM signals in [4]. It should be noted that the DFT is able to provide the circular convolution property, significantly simplifying the channel equalization in frequency domain, which, however, is not shared with the DCT. On the other hand, recalling that the DCT-OFDM equalizers are developed for linear time-invariant channels, its performance cannot be guaranteed for time-varying channels.

Through exploiting cyclic prefix (CP), a low-complexity one-tap equalizer can be devised to efficiently eliminate the inter-symbol interference (ISI) for OFDM systems in quasi-static channels provided that the CP length is not less than the channel length. Unlike the quasi-static channels, however, the timevarying channels destroy the subchannel orthogonality, incurring intercarrier interference (ICI) and eventually resulting in a serious degradation in system performance. In order to overcome the ICI effect, various methodologies such as differential coding [5], ICI self-cancellation [6], Doppler diversity [7] and ICI cancellation-based equalization [8-24], have been proposed. Among them, the ICI cancellation-based equalization approach is the most promising because of its good performance under the situation of perfect channel estimation.

The ICI cancellation-based equalizers can be classified as linear and nonlinear approaches. The linear solutions, such as the least squares (LS) [8] and 
minimum mean square error (MMSE) [9-11] equalizers, are of computational simplicity. As a result, they have received much attention in the literature. However, for time-varying channels, the above linear equalizers cannot efficiently exploit the diversity in time domain, suffering serious performance degradation. To circumvent this issue, widely linear (WL) filtering techniques [12-15] and non-linear equalizers [16-24] have been suggested.

The WL equalizers exploit the non-circularity of the received data to obtain extra degrees-of-freedom (DoFs) for ICI cancellation. In order to implement the OFDM signal detection, two complicated equalization matrices inherent in the WL approaches need to be determined, requiring very high computational complexity. As a non-linear equalizer, the MMSE with successive detection (MMSE-SD) approach [16] employs decision feedback or ICI cancellation in the detection procedure. However, when the number of subcarriers in an OFDM symbol increases, their computational loads also increase rapidly. Actually, it is shown in [17-20] that the small ICI coefficients in channel matrix can be 50 ignored so that the computational cost is significantly reduced. Nevertheless, this strategy usually suffers performance degradation because the ICI coefficients are not equal to zero. Recently, a space alternating generalized expectationmaximisation (SAGE) technique has been developed to solve the signal detection problem in [21]. It has been revealed that the SAGE method is able to provide not only low computational complexity but also superior detection performance.

In order to efficiently suppress the ICI, additional information of the wireless systems, such as the CP, has been exploited to improve the signal detection methodologies. Usually, the length of CP should not be less than the maximum propagation delay of users so that the receiver is able to operate properly even 60 in worst environment. It is shown in [22] that user delay spreads are usually much less than the CP length so that their partial CP is ISI-free in many practical scenarios.. Based on this phenomenon, the ISI-free part of a CP and its corresponding OFDM signal are linearly combined to soothe the ICI effect [22]. However, it is difficult to determine which part of CP is ISI-free when the 65 maximum propagation delay is unknown. 
As is well known, the maximum likelihood (ML) algorithm is optimal in terms of ICI cancellation for OFDM signal detection, but it requires prohibitively computational complexity. To address this drawback, ML variants with less computations have been proposed [23, 24]. In [23], the OFDM signal detection 70 is first formulated as an integer least squares (ILS) problem. Then, the sequential probabilistic integer least squares (SPILS) and quick SPILS (QSPILS) are applied to obtain the transmitted data. However, since the a posteriori probability of transmitted data vector is estimated via matrix factorization, it is inaccurate when the number of subcarriers becomes large. On the other hand, 75 [24] devises a robust approach under the variational inference framework which achieves a low bit-error-rate (BER) performance even when the non-uniform noise is present.

As a low-order modulation, BPSK has been widely adopted in many existing communication protocols, such as IEEE 802.11a [25] and the second generation so digital terrestrial television broadcasting system [26], to guarantee the quality of service (QoS) against poor wireless channels. Nevertheless, the aforementioned methods ignore the fact that the BPSK-OFDM signals are real-valued, which, when properly used, can further enhance the detection performance. In this work, the real-valued property of BPSK-OFDM signals is employed to devise an ${ }_{85}$ accurate real-valued MMSE (RV-MMSE) algorithm for linear equalization. The performance analysis reveals that the RV-MMSE method is capable of achieving $3 \mathrm{~dB}$ gain over the state-of-the-art linear equalizers. To efficiently utilize the diversity of time-varying channels, the RV-MMSE approach is extended to the non-linear equalization by adopting the SD strategy, ending up with a RV90 MMSE-SD method for non-linear equalization. As the RV-MMSE-SD scheme fully utilizes the real-valued property of BPSK-OFDM signal and avoids matrix inversion in the SD iteration, it provides more accurate detection and lower computational complexity than the existing non-linear equalizers. It is worth pointing out that, the proposed interference cancellation approach can also be 95 applied to other systems with complex-valued constellation and MIMO-OFDM systems when the complex-valued system model is switched to the real-valued 
system model [20].

The rest of the paper is organized as follows. Section 2 introduces the system model. Section 3 presents the RV-MMSE method. Performance analysis is conducted in Section 4. The RV-MMSE algorithm is extended to the nonlinear equalization in Section 5. The computational complexities of the proposed methods are analyzed in Section 6. Simulation results are presented in Section 7. Finally, conclusions are drawn in Section 8.

Throughout the paper, boldface lowercase and uppercase letters are used for vectors and matrices, respectively. Superscripts ${ }^{T},{ }^{*},{ }^{H}$, and ${ }^{-1}$ denote transpose, conjugate, conjugate transpose and inversion, respectively. The $\mathbb{E}[\cdot]$ represents mathematical expectation while $\operatorname{tr}(\cdot)$ is the trace operator. The $\mathbf{F}$ denotes the DFT matrix and $\mathbf{F}^{H}$ stands for the inverse DFT (IDFT) matrix. Furthermore, $\mathbf{e}_{i}$ is an $N \times 1$ vector with the $i$-th entry being one while elsewhere zero, $\mathbf{I}_{N}$ and $\mathbf{0}_{M \times N}$ are the $N \times N$ identity matrix and the $M \times N$ zero matrix, respectively.

\section{System Model}

The $n$-th transmitted sample of an OFDM symbol with a CP of length $G(G \geq L)$ can be expressed as

$$
x(n)=\frac{1}{N} \sum_{k=0}^{N-1} d(k) e^{j 2 \pi k n / N}, \quad-G \leq n \leq N-1
$$

where $L$ is the number of channel taps, $N$ is the number of subcarriers, $d(k)$ is the transmitted symbol in frequency domain at the $k$-th subcarrier and $j=\sqrt{-1}$. Without loss of generality, we assume that the transmitted symbols are zero mean, i.e., $\mathbb{E}[d(k)]=0$, and uncorrelated with each other, i.e., $\mathbb{E}\left[d(i) d^{*}(j)\right]=0$ for $i \neq j$. Furthermore, it is assumed that frequency and time synchronizations have been achieved. After removing the $\mathrm{CP}$, the received signal is

$$
u(n)=\sum_{l=0}^{L-1} h_{n}^{l} x(n-l)+w(n), \quad 0 \leq n \leq N-1
$$


where $h_{n}^{l}$ is the $l$-th channel tap in the $n$-th sample of the OFDM symbol and $w(n)$ denotes the additive noise which is assumed to be zero-mean circularly symmetric complex Gaussian (ZMCSCG) distributed.

Collecting the samples of the received signal into a vector, i.e., $\mathbf{u}=[u(0), \cdots, u(N-1)]^{T}$, it follows from (2) that

$$
\mathbf{u}=\breve{\mathbf{H}} \mathbf{x}+\mathbf{w}
$$

where $\mathbf{x}=[x(0), \cdots, x(N-1)]^{T}, \mathbf{w}=[w(0), \cdots, u(N-1)]^{T}$ and $\breve{\mathbf{H}}$ are the transmitted signal vector, noise vector and an $N \times N$ channel impulse response matrix in time domain, respectively. Using $h_{n}^{l}=0$ for $N>l \geq L$, the channel matrix $\breve{\mathbf{H}}$ for the $n$-th sample and $l$-th tap can be expressed as

$$
\breve{\mathbf{H}}(n, l)=h_{n}^{<n-l>_{N}}
$$

where $\langle\cdot\rangle_{N}$ denotes the modulo- $N$ operator.

Taking DFT on (3), we have

$$
\begin{aligned}
\mathbf{r} & \triangleq \mathbf{F u} \\
& =\mathbf{F} \breve{H} \mathbf{x}+\mathbf{F} \mathbf{w} \\
& =\mathbf{F} \breve{H} \mathbf{F}^{H} \mathbf{F} \mathbf{x}+\mathbf{F w} \\
& \triangleq \mathbf{H} \mathbf{d}+\mathbf{v}
\end{aligned}
$$

where $\mathbf{d}=\mathbf{F x}$ and $\mathbf{v}=\mathbf{F w}$ are the $N$-point DFTs of $\mathbf{x}$ and $\mathbf{w}$, respectively, while $\mathbf{H}=\mathbf{F} \mathbf{H} \mathbf{F}^{H} \in \mathbb{C}^{N \times N}$ is the channel impulse response matrix in frequency domain. Unlike the quasi-static channels, as the gain of each path is not unchanged during the time duration of one OFDM symbol, $\breve{\mathbf{H}}$ is not a circular Toeplitz matrix so that $\mathbf{H}$ is no longer a diagonal matrix. The off-diagonal elements in $\mathbf{H}$ thus result in ICI, incurring a serious degradation in system performance.

It is assumed that $\mathbf{H}$, which can be obtained by pilot-assisted channel estimation methods $[27,28]$, is available at the receiver. The task in this work is to find the signal $\mathbf{d}$ using the received data $\mathbf{r}$. 


\section{RV-MMSE Equalizer}

In this section, we propose a linear equalizer without decision feedback for BPSK-OFDM systems. We will first briefly review the traditional MMSE equalizer, revealing that the a priori information, namely, the transmitted data are real-valued, is neglected. Then, the proposed RV-MMSE equalizer, which makes full use of the real-valued property of the transmitted data, is presented.

\subsection{Traditional MMSE Equalizer}

It is well known that the MMSE equalizer, which minimizes the total powers of the ICI component and Gaussian noise at the output of the receiver, usually outperforms the LS equalizer. According to the MMSE criterion, an equalization matrix is firstly constructed. The transmitted signal $\mathbf{d}$ can be estimated by leftmultiplying $\mathbf{r}$ by an equalization matrix $\boldsymbol{\Omega}[16]$ :

$$
\hat{\mathbf{d}}_{\mathrm{MMSE}}=\mathbf{\Omega r}
$$

where

$$
\boldsymbol{\Omega}=\mathbf{H}^{H}\left(\mathbf{H H}^{H}+\frac{\sigma_{\mathrm{v}}^{2}}{\sigma_{\mathrm{d}}^{2}} \mathbf{I}_{N}\right)^{-1}
$$

with $\sigma_{\mathbf{v}}^{2}$ and $\sigma_{\mathbf{d}}^{2}$ being the variances of the elements of $\mathbf{v}$ and $\mathbf{d}$, respectively.

Note that the traditional MMSE equalizer is applicable to various modulation types in OFDM systems, such as the M-ary quadrature amplitude modulation (MQAM) and M-ary phase shift keying (MPSK). However, the transmitted signals in the BPSK-OFDM systems are also taken as complex-valued in the classical MMSE equalizer. Unlike the existing MMSE scheme, the RVMMSE approach will be devised by considering the real-valued property of BPSK-OFDM signal.

\subsection{RV-MMSE Equalizer}

As $\mathbf{d}$ is real-valued, the real and imaginary components of $\mathbf{r}$ can be expressed as

$$
\Re\{\mathbf{r}\}=\Re\{\mathbf{H}\} \mathbf{d}+\Re\{\mathbf{v}\}
$$


and

$$
\Im\{\mathbf{r}\}=\Im\{\mathbf{H}\} \mathbf{d}+\Im\{\mathbf{v}\} .
$$

It follows from (5) that

$$
\mathbf{y}=\mathbf{Q d}+\mathbf{n}
$$

where

$$
\mathbf{y}=\left[\Re\{\mathbf{r}\}^{T} \quad \Im\{\mathbf{r}\}^{T}\right]^{T}
$$

145

$$
\begin{gathered}
\mathbf{Q}=\left[\begin{array}{ll}
\Re\{\mathbf{H}\}^{T} & \Im\{\mathbf{H}\}^{T}
\end{array}\right]^{T} \\
\mathbf{n}=\left[\begin{array}{ll}
\Re\{\mathbf{v}\}^{T} & \Im\{\mathbf{v}\}^{T}
\end{array}\right]^{T} .
\end{gathered}
$$

It is interesting to observe from (9) that, via employing the real-valued property of BPSK signals, we are able to increase the data observation length from $N$ to $2 N$ without destroying the structure of the transmitted data $\mathbf{d}$, providing more DoFs to suppress the noise. As the estimated parameter vector has a linear relationship of $\mathbf{y}$, we obtain

$$
\hat{\mathbf{d}}_{\mathrm{RV}-\mathrm{MMSE}}=\hat{\mathbf{G}} \mathbf{y}
$$

where $\hat{\mathbf{G}}$ is the estimate of the unknown equalization matrix $\mathbf{G} \in \mathbb{R}^{N \times 2 N}$, which can be determined as

$$
\hat{\mathbf{G}}=\arg \min _{\mathbf{G}} \mathbb{E}\left[\|\mathbf{d}-\mathbf{G y}\|^{2}\right]
$$

where $\|\cdot\|$ stands for Euclidean norm. To this end, by setting the first derivative of $\mathbb{E}\left[\|\mathbf{d}-\mathbf{G y}\|^{2}\right]$ with respect to $\mathbf{G}$ to zero, we have

$$
\begin{aligned}
\frac{\partial}{\partial \mathbf{G}}\left\{\mathbb{E}\left[(\mathbf{d}-\mathbf{G y})^{T}(\mathbf{d}-\mathbf{G y})\right]\right\} & =\mathbb{E}\left\{\frac{\partial}{\partial \mathbf{G}}\left[\operatorname{tr}\left((\mathbf{d}-\mathbf{G y})(\mathbf{d}-\mathbf{G y})^{T}\right)\right]\right\} \\
& =2 \mathbb{E}\left[-\mathbf{d y}^{T}+\mathbf{G y y}^{T}\right] \\
& =\mathbf{0}_{N \times 2 N} .
\end{aligned}
$$


Since the transmitted vector $\mathbf{d}$ is uncorrelated with the ZMCSCG vector $\mathbf{v}$, i.e., $\mathbb{E}\left[\mathbf{d}^{T} \mathbf{v}\right]=0$, substituting (9) into (12) yields

$$
\begin{aligned}
& \mathbb{E}\left[\mathbf{G}(\mathbf{Q d}+\mathbf{n})(\mathbf{Q d}+\mathbf{n})^{T}\right]-\mathbb{E}\left[\mathbf{d}(\mathbf{Q d}+\mathbf{n})^{T}\right] \\
= & \mathbf{G}\left(\mathbf{Q} \mathbb{E}\left[\mathbf{d d}^{T}\right] \mathbf{Q}^{T}+\mathbb{E}\left[\mathbf{n n}^{T}\right]\right)-\mathbb{E}\left[\mathbf{d} \mathbf{d}^{T}\right] \mathbf{Q}^{T} \\
= & \mathbf{0}_{N \times 2 N} .
\end{aligned}
$$

This leads to

$$
\hat{\mathbf{G}}=\mathbf{Q}^{T}\left(\mathbf{Q} \mathbf{Q}^{T}+\frac{\sigma_{\mathbf{n}}^{2}}{\sigma_{\mathbf{d}}^{2}} \mathbf{I}_{2 N}\right)^{-1}
$$

where $\sigma_{\mathbf{n}}^{2}$ denotes the variance of $\mathbf{n}$.

As $\mathbf{v}$ is the ZMCSCG vector, its real component has the same power as its imaginary component, i.e., $\sigma_{\mathbf{n}}^{2}=\frac{1}{2} \sigma_{\mathbf{v}}^{2}$. Substituting this equality into (14) yields

$$
\hat{\mathbf{G}}=\mathbf{Q}^{T}\left(\mathbf{Q} \mathbf{Q}^{T}+\frac{\sigma_{\mathbf{v}}^{2}}{2 \sigma_{\mathbf{d}}^{2}} \mathbf{I}_{2 N}\right)^{-1} .
$$

Since $\mathbf{Q} \mathbf{Q}^{T}+\sigma_{\mathbf{v}}^{2} /\left(2 \sigma_{\mathbf{d}}^{2}\right) \mathbf{I}_{2 N}$ has the size of $2 N \times 2 N$, its inversion is more computationally demanding compared to (6). According to the matrix identity $\mathbf{A}(\mathbf{I}+\mathbf{B A})^{-\mathbf{1}} \equiv(\mathbf{I}+\mathbf{A B})^{-\mathbf{1}} \mathbf{A}[29]$, (15) can be rewritten as

$$
\hat{\mathbf{G}}=\left(\mathbf{Q}^{T} \mathbf{Q}+\frac{\sigma_{\mathbf{v}}^{2}}{2 \sigma_{\mathbf{d}}^{2}} \mathbf{I}_{N}\right)^{-1} \mathbf{Q}^{T}
$$

Apparently, $\mathbf{Q}^{T} \mathbf{Q}+\sigma_{\mathbf{v}}^{2} /\left(2 \sigma_{\mathbf{d}}^{2}\right) \mathbf{I}_{N}$ is an $N \times N$ matrix, enabling us to calculate G with lower computational requirement than (15).

\section{Performance Analysis}

In this section, we will prove that the proposed RV-MMSE equalizer is able to provide a better performance than the traditional MMSE equalizer when the signal-to-noise ratio (SNR) is sufficiently high.

Substituting (16) into (10), the $i$-th subcarrier information can be obtained 
by

$$
\begin{aligned}
\hat{d}_{i} & =\mathbf{e}_{i}^{T}\left(\mathbf{Q}^{T} \mathbf{Q}+\frac{\sigma_{\mathbf{v}}^{2}}{2 \sigma_{\mathbf{d}}^{2}} \mathbf{I}_{N}\right)^{-1} \mathbf{Q}^{T} \mathbf{y} \\
& =\mathbf{e}_{i}^{T}\left(\mathbf{Q}^{T} \mathbf{Q}+\frac{\sigma_{\mathbf{v}}^{2}}{2 \sigma_{\mathbf{d}}^{2}} \mathbf{I}_{N}\right)^{-1} \mathbf{Q}^{T} \mathbf{Q} \mathbf{d}+\mathbf{e}_{i}^{T}\left(\mathbf{Q}^{T} \mathbf{Q}+\frac{\sigma_{\mathbf{e}}^{2}}{2 \sigma_{\mathbf{d}}^{2}} \mathbf{I}_{N}\right)^{-1} \mathbf{Q}^{T} \mathbf{n} \\
& =\underbrace{\mathbf{e}_{i}^{T}\left(\mathbf{Q}^{T} \mathbf{Q}+\frac{\sigma_{\mathbf{v}}^{2}}{2 \sigma_{\mathbf{d}}^{2}} \mathbf{I}_{N}\right)^{-1} \mathbf{Q}^{T} \mathbf{Q} \mathbf{e}_{i} d_{i}}_{a_{i}} \\
+ & \underbrace{\sum_{j \neq i} \mathbf{e}_{i}^{T}\left(\mathbf{Q}^{T} \mathbf{Q}+\frac{\sigma_{\mathbf{v}}^{2}}{2 \sigma_{\mathbf{d}}^{2}} \mathbf{I}_{N}\right)^{-1} \mathbf{Q}^{T} \mathbf{Q} \mathbf{e}_{j} d_{j}+\mathbf{e}_{i}^{T}\left(\mathbf{Q}^{T} \mathbf{Q}+\frac{\sigma_{\mathbf{v}}^{2}}{2 \sigma_{\mathbf{d}}^{2}} \mathbf{I}_{N}\right)^{-1} \mathbf{Q}^{T} \mathbf{n}}_{\psi_{i}}
\end{aligned}
$$

where $a_{i}$ and $\psi_{i}$ are the desired signal and sum of the residual ICI and noise, respectively. In order to evaluate the performance of the RV-MMSE equalizer, the post-processing signal-to-interference-plus-noise ratio (PPSINR), which is regarded as a good indicator for the BER performance of communication systems, will be examined.

According to (17), the PPSINR of the $i$-th subcarrier for the RV-MMSE equalizer can be calculated as

$$
\gamma_{i}=\frac{\operatorname{var}\left(a_{i}\right)}{\operatorname{var}\left(\psi_{i}\right)}
$$

where $\operatorname{var}\left(a_{i}\right)$ and $\operatorname{var}\left(\psi_{i}\right)$ represent the variances of $a_{i}$ and $\psi_{i}$, respectively. The calculations of $\operatorname{var}\left(a_{i}\right)$ and $\operatorname{var}\left(\psi_{i}\right)$ are provided as follows.

\subsection{Computation of $\operatorname{var}\left(a_{i}\right)$}

Recalling that the transmitted signals have zero mean, $\operatorname{var}\left(a_{i}\right)$ can be expressed as

$$
\begin{aligned}
\operatorname{var}\left(a_{i}\right) & =\sigma_{\mathbf{d}}^{2} \mathbf{e}_{i}^{T}\left(\mathbf{Q}^{T} \mathbf{Q}+\frac{\sigma_{\mathbf{v}}^{2}}{2 \sigma_{\mathbf{d}}^{2}} \mathbf{I}_{N}\right)^{-1} \mathbf{Q}^{T} \mathbf{Q} \mathbf{e}_{i} \times \mathbf{e}_{i}^{T} \mathbf{Q}^{T} \mathbf{Q}\left(\mathbf{Q}^{T} \mathbf{Q}+\frac{\sigma_{\mathbf{v}}^{2}}{2 \sigma_{\mathbf{d}}^{2}} \mathbf{I}_{N}\right)^{-1} \mathbf{e}_{i} \\
& =\sigma_{\mathbf{d}}^{2}\left[\mathbf{e}_{i}^{T}\left(\mathbf{Q}^{T} \mathbf{Q}+\frac{\sigma_{\mathbf{v}}^{2}}{2 \sigma_{\mathbf{d}}^{2}} \mathbf{I}_{N}\right)^{-1} \mathbf{Q}^{T} \mathbf{Q} \mathbf{e}_{i}\right]^{2}
\end{aligned}
$$

It is shown in Appendix A that, as SNR becomes high, we have

$$
\mathbf{e}_{i}^{T}\left(\mathbf{Q}^{T} \mathbf{Q}+\frac{\sigma_{\mathbf{v}}^{2}}{2 \sigma_{\mathbf{d}}^{2}} \mathbf{I}_{N}\right)^{-1}\left(\mathbf{Q}^{T} \mathbf{Q}\right) \mathbf{e}_{i} \approx 1-\frac{\sigma_{\mathbf{v}}^{2}}{2 \sigma_{\mathbf{d}}^{2}} \mathbf{e}_{i}^{T}\left(\mathbf{Q}^{T} \mathbf{Q}\right)^{-1} \mathbf{e}_{i}
$$


Substituting (20) into (19) yields

$$
\begin{aligned}
\operatorname{var}\left(a_{i}\right) & \approx \sigma_{\mathbf{d}}^{2}\left(1-\frac{\sigma_{\mathbf{v}}^{2}}{2 \sigma_{\mathbf{d}}^{2}} \mathbf{e}_{i}^{T}\left(\mathbf{Q}^{T} \mathbf{Q}\right)^{-1} \mathbf{e}_{i}\right)^{2} \\
& =\sigma_{\mathbf{d}}^{2}\left(1-\frac{\sigma_{\mathbf{v}}^{2}}{\sigma_{\mathbf{d}}^{2}} \mathbf{e}_{i}^{T}\left(\mathbf{Q}^{T} \mathbf{Q}\right)^{-1} \mathbf{e}_{i}+\left(\frac{\sigma_{\mathbf{v}}^{2}}{2 \sigma_{\mathbf{d}}^{2}}\right)^{2}\left(\mathbf{e}_{i}^{T}\left(\mathbf{Q}^{T} \mathbf{Q}\right)^{-1} \mathbf{e}_{i}\right)^{2}\right) \\
& \approx \sigma_{\mathbf{d}}^{2}\left(1-\frac{\sigma_{\mathbf{v}}^{2}}{\sigma_{\mathbf{d}}^{2}} \mathbf{e}_{i}^{T}\left(\mathbf{Q}^{T} \mathbf{Q}\right)^{-1} \mathbf{e}_{i}\right)
\end{aligned}
$$

Note that the approximation in (21c) is valid because the second term $\left(\sigma_{\mathbf{v}}^{2} /\left(2 \sigma_{\mathbf{d}}^{2}\right)\right)^{2}\left(\mathbf{e}_{i}^{T}\left(\mathbf{Q}^{T} \mathbf{Q}\right)^{-1} \mathbf{e}_{i}\right)^{2}$ in $(21 b)$ is much smaller than other terms.

\subsection{Derivation of $\operatorname{var}\left(\psi_{i}\right)$}

As the transmitted signals are uncorrelated, we have

$$
\operatorname{var}\left(\psi_{i}\right)=\operatorname{var}\left(\hat{d}_{i}\right)-\operatorname{var}\left(a_{i}\right)
$$

Similar to $(19), \operatorname{var}\left(\hat{d}_{i}\right)$ can be expressed as

$$
\begin{aligned}
\operatorname{var}\left(\hat{d}_{i}\right) & =\mathbb{E}\left[\hat{d}_{i} \hat{d}_{i}^{T}\right] \\
& =\mathbb{E}\left[\mathbf{e}_{i}^{T} \mathbf{Q}^{T}\left(\mathbf{Q} \mathbf{Q}^{T}+\frac{\sigma_{\mathbf{v}}^{2}}{2 \sigma_{\mathbf{d}}^{2}} \mathbf{I}_{2 N}\right)^{-1} \mathbf{y} \mathbf{y}^{T} \mathbf{Q}\left(\mathbf{Q}^{T} \mathbf{Q}+\frac{\sigma_{\mathbf{v}}^{2}}{2 \sigma_{\mathbf{d}}^{2}} \mathbf{I}_{N}\right)^{-1} \mathbf{e}_{i}\right] \\
& =\mathbf{e}_{i}^{T} \mathbf{Q}^{T}\left(\mathbf{Q} \mathbf{Q}^{T}+\frac{\sigma_{\mathbf{v}}^{2}}{2 \sigma_{\mathbf{d}}^{2}} \mathbf{I}_{2 N}\right)^{-1} \mathbb{E}\left[(\mathbf{Q} \mathbf{d}+\mathbf{n})(\mathbf{Q d}+\mathbf{n})^{T}\right] \mathbf{Q}\left(\mathbf{Q}^{T} \mathbf{Q}+\frac{\sigma_{\mathbf{v}}^{2}}{2 \sigma_{\mathbf{d}}^{2}} \mathbf{I}_{N}\right)^{-1} \mathbf{e}_{i} \\
& =\sigma_{\mathbf{d}}^{2} \mathbf{e}_{i}^{T} \mathbf{Q}^{T} \mathbf{Q}\left(\mathbf{Q}^{T} \mathbf{Q}+\frac{\sigma_{\mathbf{v}}^{2}}{2 \sigma_{\mathbf{d}}^{2}} \mathbf{I}_{N}\right)^{-1} \mathbf{e}_{i} \\
& \approx \sigma_{\mathbf{d}}^{2}\left(1-\frac{\sigma_{\mathbf{v}}^{2}}{2 \sigma_{\mathbf{d}}^{2}} \mathbf{e}_{i}^{T}\left(\mathbf{Q}^{T} \mathbf{Q}\right)^{-1} \mathbf{e}_{i}\right)
\end{aligned}
$$

Substituting (21c) and (23) into $(22)$, var $\left(\psi_{i}\right)$ can be further evaluated as

$$
\begin{aligned}
\operatorname{var}\left(\psi_{i}\right) & \approx \sigma_{\mathbf{d}}^{2}\left(1-\frac{\sigma_{\mathbf{v}}^{2}}{2 \sigma_{\mathbf{d}}^{2}} \mathbf{e}_{i}^{T}\left(\mathbf{Q}^{T} \mathbf{Q}\right)^{-1} \mathbf{e}_{i}\right)-\sigma_{\mathbf{d}}^{2}\left(1-\frac{\sigma_{\mathbf{v}}^{2}}{\sigma_{\mathbf{d}}^{2}} \mathbf{e}_{i}^{T}\left(\mathbf{Q}^{T} \mathbf{Q}\right)^{-1} \mathbf{e}_{i}\right) \\
& =\frac{\sigma_{\mathbf{v}}^{2}}{2} \mathbf{e}_{i}^{T}\left(\left(\mathbf{Q}^{T} \mathbf{Q}\right)^{-1}\right) \mathbf{e}_{i} .
\end{aligned}
$$

In order to illustrate the superiority of the RV-MMSE equalizer over the traditional one in terms of PPSINR, the variances of $\bar{a}_{i}$ and $\bar{\psi}_{i}$ for the traditional MMSE equalizer are also needed. By adopting similar manipulations, they can be determined, respectively, as

$$
\operatorname{var}\left(\bar{a}_{i}\right)=\sigma_{\mathbf{d}}^{2}\left(1-2 \frac{\sigma_{\mathbf{v}}^{2}}{\sigma_{\mathbf{d}}^{2}} \mathbf{e}_{i}{ }^{H}\left(\mathbf{H}^{H} \mathbf{H}\right)^{-1} \mathbf{e}_{i}\right)
$$


and

$$
\operatorname{var}\left(\bar{\psi}_{i}\right)=\sigma_{\mathbf{v}}^{2} \mathbf{e}_{i}^{T}\left(\mathbf{H}^{H} \mathbf{H}\right)^{-1} \mathbf{e}_{i} .
$$

However, it is difficult to directly determine the PPSINRs for the RV-MMSE and traditional MMSE equalizers. Alternatively, we can examine the superiority of the RV-MMSE equalizer over the traditional MMSE equalizer by qualitatively comparing their PPSINRs. To this end, substituting (21c) and (24) into (18), we get

$$
\begin{aligned}
\gamma_{i} & =\frac{\sigma_{\mathbf{d}}^{2}\left(1-\left(\sigma_{\mathbf{v}}^{2} / \sigma_{\mathbf{d}}^{2}\right) \mathbf{e}_{i}^{T}\left(\mathbf{Q}^{T} \mathbf{Q}\right)^{-1} \mathbf{e}_{i}\right)}{\left(\sigma_{\mathbf{v}}^{2} / 2\right) \mathbf{e}_{i}^{T}\left(\mathbf{Q}^{T} \mathbf{Q}\right)^{-1} \mathbf{e}_{i}} \\
& =2\left(\frac{\sigma_{\mathbf{d}}^{2}}{\sigma_{\mathbf{v}}^{2}} \frac{1}{\mathbf{e}_{i}^{T}\left(\mathbf{Q}^{T} \mathbf{Q}\right)^{-1} \mathbf{e}_{i}}-1\right) .
\end{aligned}
$$

Similarly, employing (25) and (26), the PPSINR of the $i$-th subcarrier for the traditional MMSE equalizer can be obtained as

$$
\begin{aligned}
\bar{\gamma}_{i} & =\frac{\sigma_{\mathbf{d}}^{2}\left(1-2\left(\sigma_{\mathbf{v}}^{2} / \sigma_{\mathbf{d}}^{2}\right) \mathbf{e}_{i}^{T}\left(\mathbf{H}^{T} \mathbf{H}\right)^{-1} \mathbf{e}_{i}\right)}{\sigma_{\mathbf{v}}^{2} \mathbf{e}_{i}^{T}\left(\mathbf{H}^{T} \mathbf{H}\right)^{-1} \mathbf{e}_{i}} \\
& =\frac{\sigma_{\mathbf{d}}^{2}}{\sigma_{\mathbf{v}}^{2}} \frac{1}{\mathbf{e}_{i}^{T}\left(\mathbf{H}^{T} \mathbf{H}\right)^{-1} \mathbf{e}_{i}}-2 .
\end{aligned}
$$

To proceed, we need the following result.

Proposition 1. In time-varying channels and high SNR region, we have

$$
\mathbf{e}_{i}^{T}\left(\mathbf{H}^{H} \mathbf{H}\right)^{-1} \mathbf{e}_{i} \geq \mathbf{e}_{i}^{T}\left(\mathbf{Q}^{T} \mathbf{Q}\right)^{-1} \mathbf{e}_{i} .
$$

The proof of Proposition 1 is provided in Appendix B.

Therefore, it follows from (27)-(29) that

$$
\gamma_{i}>2 \bar{\gamma}_{i}
$$

This indicates that the RV-MMSE equalizer can achieve a $3 \mathrm{~dB}$ gain over the traditional one provided that SNR is sufficiently large.

It is known that channel state information is not always available in practice. Consequently, we evaluate the performance of our proposed algorithm in the presence of channel estimation error. For convenience, the channel estimation error for equalizers can be regarded as Gaussian distributed [30]. This allows 
us to evaluate our proposed algorithm by comparing the variances of postprocessing noise.

According to (17), the post-processing noise for the RV-MMSE equalizer can be represented as $b_{i}=\mathbf{e}_{i}^{T}\left(\mathbf{Q}^{T} \mathbf{Q}+\frac{\sigma_{\mathrm{v}}^{2}}{2 \sigma_{\mathrm{d}}^{2}} \mathbf{I}_{N}\right)^{-1} \mathbf{Q}^{T} \mathbf{n}$. Therefore, the variance of $b_{i}$ is calculated as

$$
\begin{aligned}
& \operatorname{var}\left(b_{i}\right)=\mathbb{E}\left[b_{i} b_{i}^{T}\right] \\
& =\frac{\sigma_{\mathbf{v}}^{2}}{2} \mathbf{e}_{i}^{T}\left(\mathbf{Q}^{T} \mathbf{Q}+\frac{\sigma_{\mathbf{v}}^{2}}{2 \sigma_{\mathbf{d}}^{2}} \mathbf{I}_{N}\right)^{-1} \mathbf{Q}^{T} \mathbf{Q}\left(\mathbf{Q}^{T} \mathbf{Q}+\frac{\sigma_{\mathbf{v}}^{2}}{2 \sigma_{\mathbf{d}}^{2}} \mathbf{I}_{N}\right)^{-1} \mathbf{e}_{i} \\
& =\sigma_{\mathbf{d}}^{2}(\frac{\sigma_{\mathbf{v}}^{2}}{2 \sigma_{\mathbf{d}}^{2}} \mathbf{e}_{i}^{T}\left(\mathbf{Q}^{T} \mathbf{Q}\right)^{-1} \mathbf{e}_{i}-\underbrace{\frac{1}{2}\left(\frac{\sigma_{\mathbf{v}}^{2}}{\sigma_{\mathbf{d}}^{2}}\right)^{2} \mathbf{e}_{i}^{T}\left(\mathbf{Q}^{T} \mathbf{Q}\right)^{-2} \mathbf{e}_{i}+\cdots}_{\text {small compared to the first term }}) \\
& \approx \frac{\sigma_{\mathrm{v}}^{2}}{2} \mathbf{e}_{i}^{T}\left(\mathbf{Q}^{T} \mathbf{Q}\right)^{-1} \mathbf{e}_{i}
\end{aligned}
$$

Similarly, the variance of post-processing noise for the traditional equalizer can be computed as

$$
\operatorname{var}\left(\bar{b}_{i}\right)=\sigma_{\mathbf{v}}^{2} \mathbf{e}_{i}^{H}\left(\mathbf{H}^{H} \mathbf{H}\right)^{-1} \mathbf{e}_{i} .
$$

With the use of $(29)$, it is easy to compare $\operatorname{var}\left(b_{i}\right)$ with $\operatorname{var}\left(\bar{b}_{i}\right)$. That is,

$$
\operatorname{var}\left(b_{i}\right) \leq \frac{1}{2} \operatorname{var}\left(\bar{b}_{i}\right)
$$

which indicates that the post-processing noise power for the RV-MMSE equalizer is at least half of that for the traditional MMSE equalizer. This in turn indicates that the RV-MMSE equalizer is more robust against channel estimation errors.

\section{RV-MMSE-SD Equalizer}

It has been pointed out in $[16,31]$ that although the time-varying channels might destroy the orthogonality of subcarriers, they provide time diversity which, when properly utilized, is able to significantly enhance the detection performance. Employing the SD technology in the MMSE-SD method [16], and the fast recursive strategy in [32], the RV-MMSE-SD approach is presented in this section, which makes good use of the time diversity for further improving the detection performance. 
To facilitate the SD procedure, we first calculate the equalization matrix in the $i$-th iteration:

$$
\hat{\mathbf{G}}^{(i)}=\left(\left(\mathbf{Q}^{(i)}\right)^{T} \mathbf{Q}^{(i)}+\frac{\sigma_{\mathbf{v}}^{2}}{2 \sigma_{\mathbf{d}}^{2}} \mathbf{I}_{N}\right)^{-1}\left(\mathbf{Q}^{(i)}\right)^{T}, i=0, \cdots, N-1
$$

where $\mathbf{Q}^{(i)}$ is the channel matrix estimate at the $i$-th iteration, which is initialized as $\mathbf{Q}^{(0)}=\mathbf{Q}$. In order to alleviate the error propagation resulted from the iterations, the equalization vector with the highest SINR needs to be picked out, whose index is determined as

$$
\begin{aligned}
\ell^{(i)} & =\arg \max _{k=1, \cdots, N} \operatorname{SINR}_{k}^{(i)} \\
& =\arg \max _{k=1, \cdots, N} \frac{\left|\left\langle\mathbf{g}_{k}^{(i)}, \mathbf{q}_{k}^{(i)}\right\rangle\right|^{2}}{\sum_{m=1, m \neq k}^{N}\left|\left\langle\mathbf{g}_{k}^{(i)}, \mathbf{q}_{m}^{(i)}\right\rangle\right|^{2}+\left(\sigma_{\mathbf{v}}^{2} / 2 \sigma_{\mathbf{d}}^{2}\right)\left\|\mathbf{g}_{k}^{(i)}\right\|^{2}}
\end{aligned}
$$

where $\langle\cdot\rangle$ denotes the inner product, $\mathbf{g}_{k}^{(i)}$ and $\mathbf{q}_{k}^{(i)}$ are the $k$-th column vectors of $\left(\hat{\mathbf{G}}^{(i)}\right)^{T}$ and $\mathbf{Q}^{(i)}$, respectively. With the so-obtained equalization vector, the $\ell^{(i)}$-th value is estimated as

$$
\hat{d}_{\ell^{(i)}}=\Upsilon\left(\left(\mathbf{g}_{\ell^{(i)}}^{(i)}\right)^{T} \mathbf{y}^{(i)}\right)
$$

where $\mathbf{y}^{(i)}$ is the real-valued observation in the last iteration and $\Upsilon(\tau)$ is a piecewise function:

$$
\Upsilon(\tau)=\left\{\begin{array}{ll}
1, & \tau \geq 0 \\
-1, & \text { else }
\end{array} .\right.
$$

To carry out the subsequent iterations, the observation vector is updated as

$$
\mathbf{y}^{(i+1)}=\mathbf{y}^{(i)}-\mathbf{q}_{\ell^{(i)}}^{(i)} \hat{d}_{\ell^{(i)}} .
$$

Meanwhile, to remove the effect of $\mathbf{q}_{\ell^{(i)}}^{(i)}$ on other subcarriers, the $\ell^{(i)}$-th column of $\mathbf{Q}^{(i)}$ needs to be set to zero, leading to

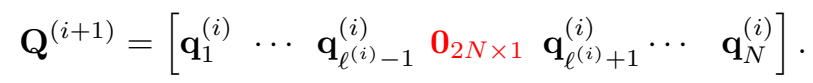

The equalization matrix can be updated by replacing $\mathbf{Q}^{(i)}$ in (34) with $\mathbf{Q}^{(i+1)}$ 190 for the $(i+1)$-th iteration. However, the matrix inversion in (34) needs to be 
conducted at each iteration, requiring $\mathcal{O}\left(7 N^{4}\right)$ real multiplications in the SD procedure [16]. This thereby leads to high computational requirement. On the other hand, it is observed from (39) that the zero column of $\mathbf{Q}^{(i+1)}$ does not provide any information for the next iteration but incurs additional computational load. To circumvent this problem, we employ the block matrix inversion and Sherman-Morrison formula to recursively update the equalization matrix.

It is observed from (35) that the $\ell^{(i)}$-th value is determined with the largest SINR, which should be removed from the observation data so that it does not affect the detection of the subsequent data. Accordingly, the channel corresponding to the $\ell^{(i)}$-th data needs to be deleted from the equalization matrix to facilitate the subsequent detection. This can be achieved by using a permutation matrix $\mathbf{P}_{\ell^{(i)}}$ to exchange the $\ell^{(i)}$-th column with the last column of $\mathbf{Q}^{(i)}$. Here, $\mathbf{P}_{\ell^{(i)}}$ is $\mathbf{I}_{N-i}$ with the $\ell^{(i)}$-th column being exchanged with the last column.

We are now at a position to recursively calculate the inverse of $\mathbf{Q}^{T} \mathbf{Q}+$ $\sigma_{\mathbf{v}}^{2} /\left(2 \sigma_{\mathbf{d}}^{2}\right) \mathbf{I}_{N}$. For simplicity, we define $\boldsymbol{\Phi}^{(i)}=\left(\tilde{\mathbf{Q}}^{(i)}\right)^{T} \tilde{\mathbf{Q}}^{(i)}+\sigma_{\mathbf{v}}^{2} /\left(2 \sigma_{\mathbf{d}}^{2}\right) \mathbf{I}_{N-i}$ for the $i$-th iteration where $\tilde{\mathbf{Q}}^{(i)}(i=0, \cdots, N-1)$ is iteratively determined with $\tilde{\mathbf{Q}}^{(0)}=\mathbf{Q}$. Moreover, we assume that $\boldsymbol{\Phi}^{(i)}$ and $\left(\boldsymbol{\Phi}^{(i)}\right)^{-1}$ have been computed at the $i$-th iteration. Using similar manipulations in [32], we partition $\Psi^{(i)} \triangleq$ $\mathbf{P}_{\ell^{(i)}} \boldsymbol{\Phi}^{(i)} \mathbf{P}_{\ell^{(i)}} \in \mathbb{R}^{(N-i) \times(N-i)}$ as

$$
\mathbf{P}_{\ell^{(i)}} \Phi^{(i)} \mathbf{P}_{\ell^{(i)}}=\left[\begin{array}{cc}
\boldsymbol{\Phi}^{(i+1)} & \mathbf{f}^{(i+1)} \\
\mathbf{f}^{(i+1)^{T}} & \zeta^{(i+1)}
\end{array}\right]
$$

where

$$
\begin{aligned}
\boldsymbol{\Phi}^{(i+1)} & =\Psi^{(i)}(1: N-i-1,1: N-i-1) \in \mathbb{R}^{(N-i-1) \times(N-i-1)} \\
\mathbf{f}^{(i+1)} & =\boldsymbol{\Psi}^{(i)}(1: N-i-1, N-i) \in \mathbb{R}^{(N-i-1) \times 1} \\
\zeta^{(i+1)} & =\Psi^{(i)}(N-i, N-i) .
\end{aligned}
$$

Here, $\boldsymbol{X}\left(c_{1}: c_{2}, r_{1}: r_{2}\right)$ stands for a submatrix constructed from row $c_{1}$ to row $c_{2}$ and from column $r_{1}$ to column $r_{2}$ of $\boldsymbol{X}$.

On the other hand, using the block matrix inversion, $\left(\Psi^{(i)}\right)^{-1} \triangleq \mathbf{P}_{\ell^{(i)}}\left(\boldsymbol{\Phi}^{(i)}\right)^{-1} \mathbf{P}_{\ell^{(i)}}$ 
can be decomposed as

$$
\left(\Psi^{(i)}\right)^{-1}=\left[\begin{array}{cc}
\left(\boldsymbol{\Gamma}^{(i+1)}\right)^{-1} & -\frac{\left(\boldsymbol{\Gamma}^{(i+1)}\right)^{-1} \mathbf{f}^{(i+1)}}{\zeta^{(i+1)}} \\
\frac{\left(\mathbf{f}^{(i+1)}\right)^{T}\left(\boldsymbol{\Gamma}^{(i+1)}\right)^{-1}}{\zeta^{(i+1)}} & \frac{1}{\zeta^{(i+1)}}+\frac{\left(\mathbf{f}^{(i+1)}\right)^{T}\left(\boldsymbol{\Gamma}^{(i+1)}\right)^{-1} \mathbf{f}^{(i+1)}}{\left(\zeta^{(i+1)}\right)^{2}}
\end{array}\right]
$$

where $\boldsymbol{\Gamma}^{(i+1)} \triangleq \boldsymbol{\Phi}^{(i+1)}-\mathbf{f}^{(i+1)}\left(\mathbf{f}^{(i+1)}\right)^{T} / \zeta^{(i+1)}$. As a result, using the ShermanMorrison formula [32], we have

$$
\begin{aligned}
\left(\mathbf{\Phi}^{(i+1)}\right)^{-1} & =\left(\boldsymbol{\Gamma}^{(i+1)}+\frac{\mathbf{f}^{(i+1)}\left(\mathbf{f}^{(i+1)}\right)^{T}}{\zeta^{(i+1)}}\right)^{-1} \\
& =\left(\boldsymbol{\Gamma}^{(i+1)}\right)^{-1}-\frac{\left(\boldsymbol{\Gamma}^{(i+1)}\right)^{-1} \mathbf{f}^{(i+1)}\left(\mathbf{f}^{(i+1)}\right)^{T}\left(\boldsymbol{\Gamma}^{(i+1)}\right)^{-1}}{\zeta^{(i+1)}+\left(\mathbf{f}^{(i+1)}\right)^{T}\left(\boldsymbol{\Gamma}^{(i+1)}\right)^{-1} \mathbf{f}^{(i+1)}}
\end{aligned}
$$

where

$$
\left(\boldsymbol{\Gamma}^{(i+1)}\right)^{-1}=\left(\Psi^{(i)}\right)^{-1}(1: N-i-1,1: N-i-1) \in \mathbb{R}^{(N-i-1) \times(N-i-1)}
$$

Thus, $\boldsymbol{\Phi}^{(i+1)}$ and $\left(\boldsymbol{\Phi}^{(i+1)}\right)^{-1}$ determined by (41) and (45), respectively, are available for the next iteration.

According to (16), we also need to update the channel matrix recursively, which can be obtained by interchanging the $\ell^{(i)}$-th column with the last column of $\tilde{\mathbf{Q}}^{(i)}$ and then deleting its last column. This eventually results into a shrunk matrix $\tilde{\mathbf{Q}}^{(i+1)}$, given by

$$
\tilde{\mathbf{Q}}^{(i+1)}=\left[\tilde{\mathbf{q}}_{1}^{(i)}, \cdots, \tilde{\mathbf{q}}_{\ell^{(i)}-1}^{(i)}, \tilde{\mathbf{q}}_{N-i}^{(i)}, \tilde{\mathbf{q}}_{\ell^{(i)}+1}^{(i)}, \cdots, \tilde{\mathbf{q}}_{N-i-1}^{(i)}\right]
$$

where $\tilde{\mathbf{q}}_{k}^{(i)}(k=1, \cdots, N)$ is the $k$-th column of $\tilde{\mathbf{Q}}^{(i)}$ in the $i$-th iteration. The RV-MMSE-SD algorithm is summarized in Algorithm 1.

\section{Complexity Requirement}

The computational complexities of the proposed algorithms are evaluated in terms of the number of real multiplications. It is worthy pointing out that the inverse of an $N \times N$ complex matrix requires $N^{3}$ complex multiplications and a complex multiplication is equivalent to three real multiplications [33]. For our proposed RV-MMSE algorithm, the main complexity lies in solving (16). The 


\footnotetext{
$\frac{\overline{\text { Algorithm } 1 \text { RV-MMSE-SD algorithm }}}{\text { Step 1: Set } i=0, \mathbf{y}^{(0)}=\mathbf{y}, \tilde{\mathbf{Q}}^{(0)}=\mathbf{Q} \text { and the index vector } \boldsymbol{\triangleq}[1, \cdots, N]^{T} .}$

Step 2: Obtain the equalization matrix by

$$
\tilde{\mathbf{G}}^{(i)}=\left(\boldsymbol{\Phi}^{(i+1)}\right)^{-1}\left(\tilde{\mathbf{Q}}^{(i)}\right)^{T}
$$

Step 3: Find the subcarrier with the highest SINR by

$$
\begin{aligned}
\ell^{(i)} & =\arg \max _{k=1, \cdots, N-i} \operatorname{SINR}_{k}^{(i)} \\
& =\arg \max _{k=1, \cdots, N-i} \frac{\left|\left\langle\tilde{\mathbf{g}}_{k}^{(i)}, \tilde{\mathbf{q}}_{k}^{(i)}\right\rangle\right|^{2}}{\sum_{m=1, m \neq k}^{N}\left|\left\langle\tilde{\mathbf{g}}_{k}^{(i)}, \tilde{\mathbf{q}}_{m}^{(i)}\right\rangle\right|^{2}+\left(\sigma_{\mathbf{v}}^{2} / 2 \sigma_{\mathbf{d}}^{2}\right)\left\|\tilde{\mathbf{g}}_{k}^{(i)}\right\|^{2}}
\end{aligned}
$$

where $\tilde{\mathbf{g}}_{k}^{(i)}$ and $\tilde{\mathbf{q}}_{k}^{(i)}$ are the $k$-th column vectors of $\left(\tilde{\mathbf{G}}^{(i)}\right)^{T}$ and $\tilde{\mathbf{Q}}^{(i)}$, respectively.

Step 4: Make a hard decision on the $\boldsymbol{\varsigma}_{\ell^{(i)}}$-th subcarrier by

$$
\hat{d}_{\boldsymbol{\varsigma}_{\ell}^{(i)}}=\Upsilon\left(\left(\tilde{\mathbf{g}}_{\ell(i)}^{(i)}\right)^{T} \mathbf{y}^{(i)}\right)
$$

where $\boldsymbol{\varsigma}_{\ell^{(i)}}$ is the $\ell^{(i)}$ entry of $\varsigma$.

Step 5: Update $\mathbf{y}^{(i+1)}$ by

$$
\mathbf{y}^{(i+1)}=\mathbf{y}^{(i)}-\tilde{\mathbf{q}}_{\ell^{(i)}}^{(i)} \hat{d}_{\boldsymbol{\varsigma}\left(\ell^{(i)}\right)} .
$$

Step 6: Determine $\left(\boldsymbol{\Phi}^{(i+1)}\right)^{-1}$ by $(45)$.

Step 7: Calculate $\tilde{\mathbf{Q}}^{(i+1)}$ by (47).

Step 9: Exchange the entries $\ell^{(i)}$ and $N-i$ of $\varsigma$.

Step 9: If $i<N-1$, set $i=i+1$ and jump to Step 2; Otherwise, stop the algorithm.

computation of $\mathbf{Q}^{T} \mathbf{Q}+\sigma_{\mathbf{v}}^{2} /\left(2 \sigma_{\mathbf{d}}^{2}\right) \mathbf{I}_{N}$ in (16) requires $4 N^{3}+N$ real multiplications, and the matrix inverse as well as matrix multiplication in (16) involve $3 N^{3}$ real multiplications. Therefore, a total of $\mathcal{O}\left(7 N^{3}\right)$ real multiplications are needed by 
the RV-MMSE approach.

The computational complexity of the RV-MMSE-SD algorithm mainly lies in (48). Since the sizes of $\Psi^{(i)}$ and $\tilde{\mathbf{Q}}^{(i)}$ decrease as $i$ increases from 0 up to $N-1$, (48) requires $2 \times N^{3}+\cdots+2 \times 1^{3}=(1 / 2)[N(N+1)]^{2}$ real multiplications. Hence, 225 the computational complexity of RV-MMSE-SD scheme only needs $\mathcal{O}\left(N^{4} / 2\right)$.

To facilitate the comparison, the computational complexities of the linear and non-linear equalizers are shown in Tables 1 and 2, respectively. In ascending order, there are RV-MMSE, LS [16], MMSE [16], RV-MMSE-SD, MMSE-SD [16] and SPILS [23] methods. Apparently, the RV-MMSE and RV-MMSE-SD algorithms are the most computationally efficient among the linear and nonlinear equalizers, respectively.

\begin{tabular}{c|c}
\multicolumn{2}{c}{ Table 1: Computational complexity of linear equalizers } \\
\hline Detection method & Number of real multiplications \\
\hline LS $[16]$ & $\mathcal{O}\left(9 N^{3}\right)$ \\
\hline MMSE $[16]$ & $\mathcal{O}\left(9 N^{3}\right)$ \\
\hline RV-MMSE & $\mathcal{O}\left(7 N^{3}\right)$ \\
\hline
\end{tabular}

Table 2: Computational complexity of non-linear equalizers

\begin{tabular}{c|c}
\hline Detection method & Number of real multiplications \\
\hline MMSE-SD [21] & $\mathcal{O}\left(8 N^{4}\right)$ \\
\hline SPILS [23] & $\mathcal{O}\left(12 N^{4}\right)$ \\
\hline RV-MMSEE-SD & $\mathcal{O}\left(N^{4} / 2\right)$ \\
\hline
\end{tabular}

Note that, in order to further reduce the computational complexity of various detection methods, the channel impulse response matrix $\mathbf{H}$ can be approximated as a banded matrix composed of the main diagonal, $p$ subdiagonals and $p$ superdiagonals of $\mathbf{H}$. The parameter $p$ can be chosen such that a trade-off is achieved between performance and complexity [17]. When the aforementioned detection methods employ the banded matrix for signal detection, the compu- 
tational load will be futher alleviated, which, however, is beyond the scope of this work. and noise of the RV-MMSE equalizer are less than half of their counterparts. Hence, based on (18), the PPSINR of the RV-MMSE equalizer is at least twice that of the MMSE equalizer when the SNR is larger than $20 \mathrm{~dB}$, which is in line 


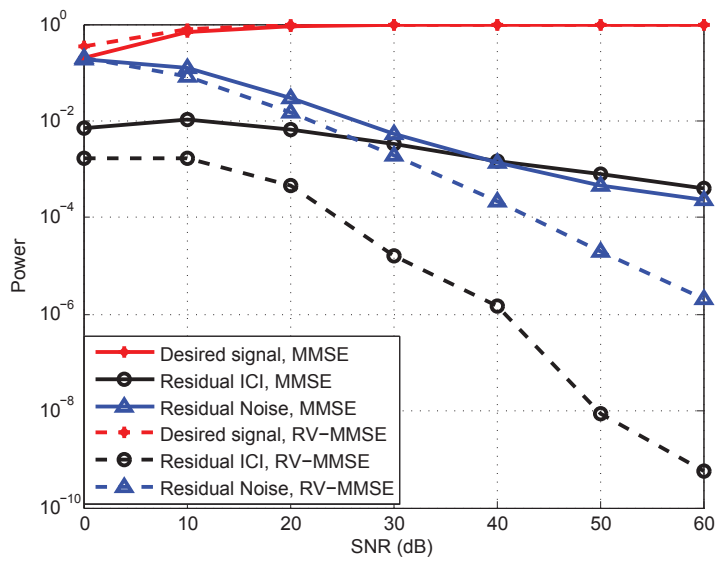

Figure 1: Powers of desired signals, residual ICI and noise for MMSE and RV-MMSE equalizers $\left(\right.$ BPSK-OFDM, $\left.f_{n}=0.1075\right)$.

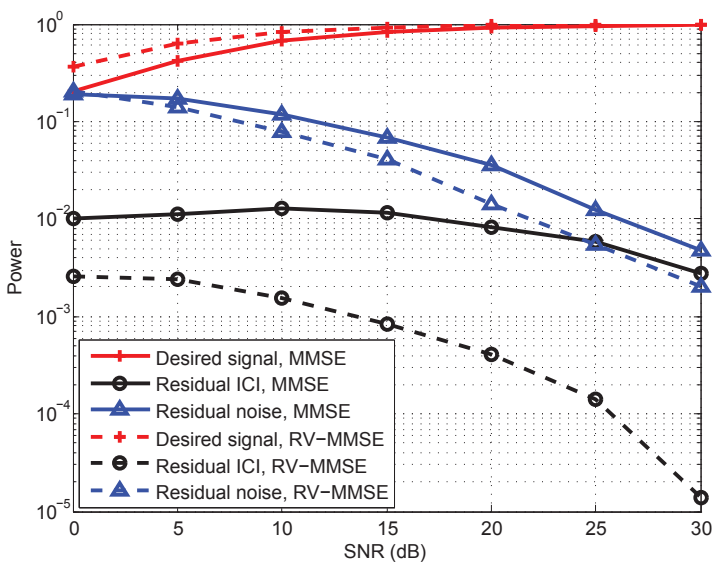

Figure 2: Powers of desired signals, residual ICI and noise for MMSE and RV-MMSE equalizers (BPSK-OFDM, $\left.f_{n}=0.26\right)$.

with the theoretical analysis in Section 4. Furthermore, Figs. 1 and 2 imply that the higher the normalized Doppler frequency is, the larger the gap between the RV-MMSE and MMSE equalizers. This in turn means that the RV-MMSE equalizer is able to get more gain at large normalized Doppler frequencies.

To compare the detection performance of our proposed algorithms with the existing approaches, the BER of BPSK-OFDM signals is plotted as a function 
of the SNR for $f_{n}=0.1075$ and $f_{n}=0.26$ in Figs. 3 and 4 , respectively. For the purpose of comparison, we also plot the matched-filter bound (MFB) [36], which is the best detection performance if the ICI is completely eliminated without noise amplification. The numerical results of the LS, MMSE, RV-MMSE, SPILS, MMSE-SD and RV-MMSE-SD equalizers are included for comparison. As expected, these algorithms can be ordered in terms of BER performance in an ascending manner: the LS, MMSE, RV-MMSE, SPILS, MMSE-SD and RV-MMSE-SD. Besides, it should be noted that the BER performance of RVMMSE-SD is very close to the MFB, validating the efficiency of the RV-MMSESD approach.

It is observed in Fig. 3 that the RV-MMSE equalizer exhibits a detection gain of about $5 \mathrm{~dB}$ over the traditional MMSE equalizer at $\mathrm{BER}=2 \times 10^{-4}$ for $f_{n}=0.1075$, while the RV-MMSE-SD equalizer is only a little bit superior to the linear equalizers in terms of BER performance. This is due to the small time diversity in slowly time-varying channels. It is seen in Fig. 4 that the RV-MMSE and RV-MMSE-SD equalizers exhibit a detection gain of about $6 \mathrm{~dB}$ over the traditional MMSE equalizer and about $1.3 \mathrm{~dB}$ over the other non-linear equalizers at $\mathrm{BER}=2 \times 10^{-4}$ for $f_{n}=0.26$, respectively. This is because the real-valued property of BPSK-OFDM signals has been utilized in the RV-MMSE and RV-MMSE-SD approaches, which, however, is ignored in other methods. Fig. 4 also indicates that the RV-MMSE-SD equalizer performs much better than its linear counterparts. This is due to the fact that it not only minimizes the powers of the residual interference and noise but also makes good use of the large time diversity in large normalized Doppler frequencies.

\subsection{Scenario 2: OFDM Signal Detection with Imperfect Channel Information}

In this subsection, we evaluate our proposed algorithms in the presence of estimated channel error. To this end, we define the normalized root mean square error (NRMSE) of the channel estimation as

$$
\operatorname{NRMSE}=\sqrt{\frac{\mathbb{E}\left[\left|h_{n}^{l}-\hat{h}_{n}^{l}\right|^{2}\right]}{\mathbb{E}\left[\left|h_{n}^{l}\right|^{2}\right]}}
$$




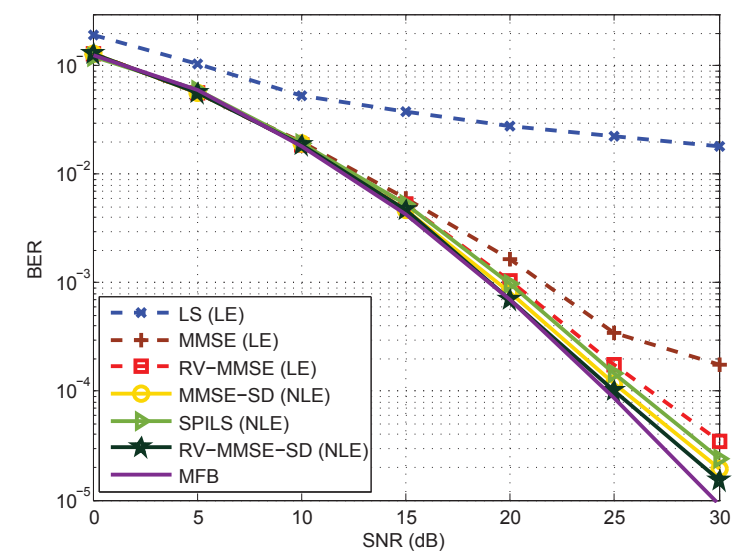

Figure 3: Performance comparison of detection algorithms (BPSK-OFDM, $f_{n}=0.1075$ ). LE and NLE stand for the linear and non-linear equalizers, respectively.

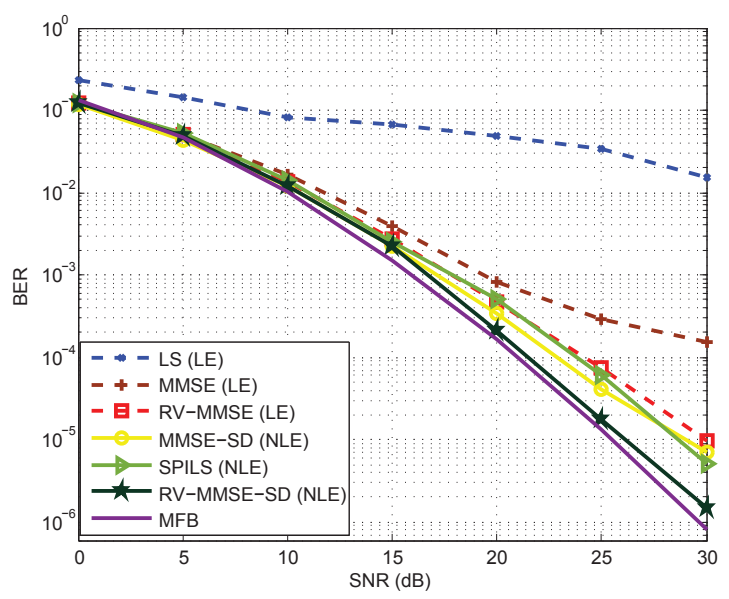

Figure 4: Performance comparison of detection algorithms (BPSK-OFDM, $f_{n}=0.26$ ).

where $\hat{h}_{n}^{l}$ represents the estimate of $h_{n}^{l}$.

Fig. 5 plots the BER performance of the MMSE, RV-MMSE and RV-MMSE$\mathrm{SD}$ for $\mathrm{NRMSE}=[0,0.05,0.1]$. As expected, the BER performance of the detection algorithms decreases as the channel estimation error becomes larger. Furthermore, Fig. 5 indicates that our proposed algorithms are relatively insensitive to the channel estimation error. This is due to the fact that the RV-MMSE and RV-MMSE-SD schemes could further eliminate the post-processing noise, 


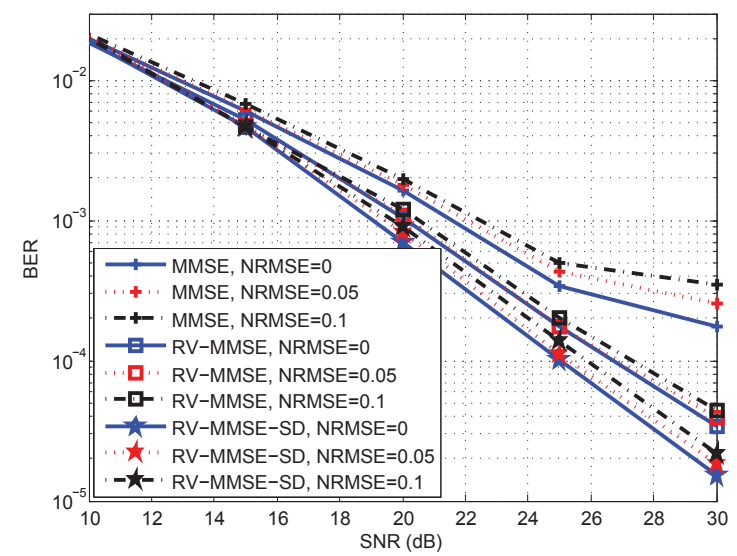

Figure 5: Detection performance for different NRMSE (BPSK-OFDM, $f_{n}=0.1075$ ).

which has been investigated in Section 4 .

\subsection{Scenario 3: Coded-OFDM Signal Detection with Perfect Channel Informa-} tion

The detection performance of OFDM signal over fading channels can be improved by introducing channel coding. Therefore, channel coding is usually adopted in most OFDM applications. In this section, we investigate the BER performance of the proposed detection algorithms for coded-OFDM systems. The coding scheme chosen for simulations is the Reed-Solomon $(255,223)$ [37].

Fig. 6 illustrates the BER performance of the LS, MMSE, SPILS, MMSESD, RV-MMSE and RV-MMSE-SD algorithms for $f_{n}=0.26$. It is observed that the superiority of our proposed approach holds also for the coded-OFDM systems. Moreover, Figs. 4 and 6 indicate that the BER gains achieved by channel coding are almost $7 \mathrm{~dB}$ at $\mathrm{BER}=2 \times 10^{-4}$ for all the algorithms except the LS method.

\section{Conclusion}

In this paper, we have devised the RV-MMSE and RV-MMSE-SD methods for transmitted signal estimation in BPSK-OFDM systems. The RV-MMSE 


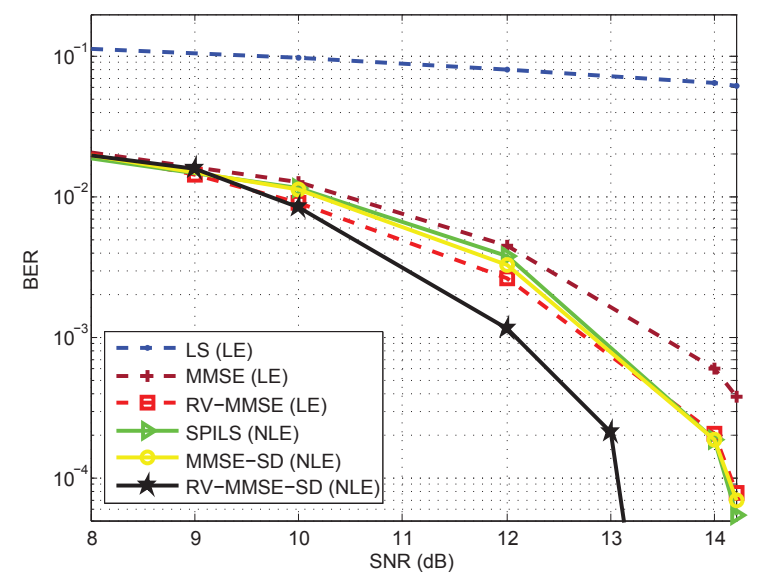

Figure 6: Detection performance of different algorithms for coded-OFDM when $f_{n}=0.26$.

algorithm considers the a priori information of the BPSK-OFDM signals in the equalization matrix construction and outperforms its traditional counterpart with lower computational complexity. In order to further improve the detection performance, the RV-MMSE-SD approach is developed, which performs the best in BPSK-OFDM signal detection among all the investigated equalizers. It is worth mentioning that the devised methods can be applied not only in the BPSK-OFDM systems but also in other complex-valued constellation and MIMO-OFDM systems.

\section{Acknowledgement}

The work described in this paper was supported by the Natural Science Foundation of China (NSFC) under Grant No. U1501253, by the Natural Science Foundation of Guangdong Province under Grant No. 2015A030311030 and by the Science and Technology Innovation Commission of Shenzhen under Grant (JCYJ20150324140036835). 


\section{Appendix A. Derivation of (20)}

It follows from $[38,39]$ that the Taylor series expansion of $\mathbf{e}_{i}^{T}\left(\mathbf{Q}^{T} \mathbf{Q}+\right.$ $\left.\sigma_{\mathbf{v}}^{2} / 2 \sigma_{\mathbf{d}}^{2} \mathbf{I}_{N}\right)^{-1}\left(\mathbf{Q}^{T} \mathbf{Q}\right)^{-1} \mathbf{e}_{i}$ is

$$
\begin{aligned}
& \mathbf{e}_{i}^{T}\left(\mathbf{Q}^{T} \mathbf{Q}+\frac{\sigma_{\mathbf{v}}^{2}}{2 \sigma_{\mathbf{d}}^{2}} \mathbf{I}_{N}\right)^{-1}\left(\mathbf{Q}^{T} \mathbf{Q}\right) \mathbf{e}_{i}=1-\underbrace{\rho \mathbf{e}_{i}^{T}\left(\mathbf{Q}^{T} \mathbf{Q}\right)^{-1} \mathbf{e}_{i}}_{\alpha_{1}} \\
& +\underbrace{\rho^{2} \mathbf{e}_{i}^{T}\left(\mathbf{Q}^{T} \mathbf{Q}\right)^{-2} \mathbf{e}_{i}}_{\alpha_{2}}-\underbrace{\rho^{3} \mathbf{e}_{i}^{T}\left(\mathbf{Q}^{T} \mathbf{Q}\right)^{-3} \mathbf{e}_{i}}_{\alpha_{3}}+\cdots
\end{aligned}
$$

where $\alpha_{1}, \alpha_{2}$ and $\alpha_{3}$ are the first-, second- and third-order terms. Applying the eigenvalue decomposition (EVD), we have $\mathbf{Q}^{T} \mathbf{Q}=\mathbf{U} \mathbf{\Lambda} \mathbf{U}^{T}$. Subsequently, (A.1) can be rewritten as

$$
\begin{aligned}
& \mathbf{e}_{i}^{T}\left(\mathbf{Q}^{T} \mathbf{Q}+\frac{\sigma_{\mathbf{v}}^{2}}{2 \sigma_{\mathbf{d}}} \mathbf{I}_{N}\right)^{-1}\left(\mathbf{Q}^{T} \mathbf{Q}\right) \mathbf{e}_{i}=1-\underbrace{\rho \sum_{j=1}^{N} \lambda_{j}^{-1} u_{i, j}^{2}}_{\alpha_{1}} \\
& +\underbrace{\rho^{2} \sum_{j=1}^{N} \lambda_{j}^{-2} u_{i, j}^{2}}_{\alpha_{2}}-\underbrace{\rho^{3} \sum_{j=1}^{N} \lambda_{j}^{-3} u_{i, j}^{2}}_{\alpha_{3}}+\cdots
\end{aligned}
$$

where $\mathbf{U}$ is composed of the eigenvectors of $\mathbf{Q}^{T} \mathbf{Q}, \boldsymbol{\Lambda}$ denotes a $N \times N$ diagonal

matrix with diagonal element equaling the eigenvalue $\lambda_{i}(i=1, \ldots, N)$ and $u_{i, j}$ represents the $(i, j)$ entry of $\mathbf{U}$. Since $\sum_{j=1}^{N} u_{i, j}^{2}=1$, the lower bound of $\alpha_{1}$ and the upper bounds of $\alpha_{2}$ and $\alpha_{3}$ can be obtained as

$$
\begin{aligned}
& \alpha_{1} \geq \rho \lambda_{\max }^{-1} \\
& \alpha_{2} \leq\left(\rho \lambda_{\min }^{-1}\right)^{2} \\
& \alpha_{3} \leq\left(\rho \lambda_{\min }^{-1}\right)^{3}
\end{aligned}
$$

where $\lambda_{\min }$ and $\lambda_{\max }$ are the minimum and maximum eigenvalues of $\mathbf{Q}^{T} \mathbf{Q}$, respectively. Thus, we have

$$
\frac{\alpha_{2}}{\alpha_{1}} \leq \frac{\left(\rho \lambda_{\min }^{-1}\right)^{2}}{\rho \lambda_{\max }^{-1}}=\frac{\rho}{\lambda_{\max }^{-1} / \lambda_{\min }^{-2}} \rightarrow 0, \text { as } \rho \rightarrow 0
$$

and

$$
\frac{\alpha_{3}}{\alpha_{1}} \leq \frac{\left(\rho \lambda_{\min }^{-1}\right)^{3}}{\rho \lambda_{\max }^{-1}}=\frac{\rho^{2}}{\lambda_{\max }^{-1} / \lambda_{\min }^{-3}} \rightarrow 0, \text { as } \rho \rightarrow 0
$$


This indicates that $\alpha_{2}$ and $\alpha_{3}$ can be ignored compared with $\alpha_{1}$, as $\mathrm{SNR}=\sigma_{\mathbf{d}}^{2} / \sigma_{\mathbf{v}}^{2}=$ $1 / 2 \rho$ is high. Therefore, we have

$$
\mathbf{e}_{i}^{T}\left(\mathbf{Q}^{T} \mathbf{Q}+\frac{\sigma_{\mathbf{v}}^{2}}{2 \sigma_{\mathbf{d}}^{2}} \mathbf{I}_{N}\right)^{-1}\left(\mathbf{Q}^{T} \mathbf{Q}\right) \mathbf{e}_{i} \approx 1-\rho \mathbf{e}_{i}^{T}\left(\mathbf{Q}^{T} \mathbf{Q}\right)^{-1} \mathbf{e}_{i}
$$

\section{Appendix B. Proof of (29)}

The $\mathbf{Q}^{T} \mathbf{Q}$ can be expanded as

$$
\begin{aligned}
\mathbf{Q}^{T} \mathbf{Q} & =\left[\begin{array}{ll}
\Re\{\mathbf{H}\}^{T} & \Im\{\mathbf{H}\}^{T}
\end{array}\right]\left[\begin{array}{c}
\Re\{\mathbf{H}\} \\
\Im\{\mathbf{H}\}
\end{array}\right] \\
& =\Re\{\mathbf{H}\}^{T} \Re\{\mathbf{H}\}+\Im\{\mathbf{H}\}^{T} \Im\{\mathbf{H}\} \\
& =\mathbf{A}
\end{aligned}
$$

where

$$
\mathbf{A}=\Re\{\mathbf{H}\}^{T} \Re\{\mathbf{H}\}+\Im\{\mathbf{H}\}^{T} \Im\{\mathbf{H}\} .
$$

Similarly, $\mathbf{H}^{H} \mathbf{H}$ is determined as

$$
\begin{aligned}
\mathbf{H}^{H} \mathbf{H} & =\left(\Re\{\mathbf{H}\}^{T}-j \Im\{\mathbf{H}\}^{T}\right)(\Re\{\mathbf{H}\}+j \Im\{\mathbf{H}\}) \\
& =\left(\Re\{\mathbf{H}\}^{T} \Re\{\mathbf{H}\}+\Im\{\mathbf{H}\}^{T} \Im\{\mathbf{H}\}\right)+j\left(\Re\{\mathbf{H}\}^{T} \Im\{\mathbf{H}\}-\Im\{\mathbf{H}\}^{T} \Re\{\mathbf{H}\}\right) \\
& =\mathbf{A}+j \mathbf{B}
\end{aligned}
$$

345 where

$$
\mathbf{B}=\Re\{\mathbf{H}\}^{T} \Im\{\mathbf{H}\}-\Im\{\mathbf{H}\}^{T} \Re\{\mathbf{H}\} .
$$

Then, we have

$$
\left(\mathbf{Q}^{T} \mathbf{Q}\right)^{-1}=\mathbf{A}^{-1}
$$

and

$$
\begin{aligned}
\left(\mathbf{H}^{H} \mathbf{H}\right)^{-1} & =(\mathbf{A}+j \mathbf{B})^{-1} \\
& =\left(\mathbf{A}+\mathbf{B} \mathbf{A}^{-1} \mathbf{B}\right)^{-1}+j \mathbf{A}^{-1} \mathbf{B}\left(\mathbf{A}+\mathbf{B} \mathbf{A}^{-1} \mathbf{B}\right)^{-1} .
\end{aligned}
$$

Since $\left(\mathbf{H}^{H} \mathbf{H}\right)^{-1}=\left(\mathbf{H}^{-1}\right)\left(\mathbf{H}^{-1}\right)^{H}, \mathbf{e}_{i}^{T}\left(\mathbf{H}^{H} \mathbf{H}\right)^{-1} \mathbf{e}_{i}$ is a real number. Therefore, we obtain

$$
\mathbf{e}_{i}^{T}(\mathbf{A}+j \mathbf{B})^{-1} \mathbf{e}_{i}=\mathbf{e}_{i}^{T}\left(\mathbf{A}+\mathbf{B} \mathbf{A}^{-1} \mathbf{B}\right)^{-1} \mathbf{e}_{i}
$$


It is shown in [18] that the ICI power is much smaller than the current subcarrier power in time-varying channels, that is, the off-diagonal elements of $\mathbf{H}$ are much smaller than the main diagonal elements of $\mathbf{H}$. Therefore, the elements in $\mathbf{B}$ are very small such that $\mathbf{B A}^{-1} \mathbf{B}$ approaches a zero matrix. Consequently, we have

$$
\left(\mathbf{A}+\mathbf{B} \mathbf{A}^{-1} \mathbf{B}\right)^{-1}=\mathbf{A}^{-1}-\mathbf{A}^{-1} \mathbf{B} \mathbf{A}^{-1} \mathbf{B} \mathbf{A}^{-1} .
$$

Moreover, it is easy to obtain $\mathbf{A}^{T}=\mathbf{A}$ and $\mathbf{B}^{T}=-\mathbf{B}$ in (B.2). Hence, the second term on the left-hand side of (B.6) can be written as

$$
\begin{aligned}
\mathbf{A}^{-1} \mathbf{B} \mathbf{A}^{-1} \mathbf{B} \mathbf{A}^{-1} & =-\mathbf{A}^{-1} \mathbf{B}\left(\mathbf{Q}^{T} \mathbf{Q}\right)^{-1} \mathbf{B}^{T}\left(\mathbf{A}^{T}\right)^{-1} \\
& =-\mathbf{A}^{-1} \mathbf{B} \mathbf{Q}^{-1}\left(\mathbf{Q}^{-1}\right)^{T} \mathbf{B}^{T}\left(\mathbf{A}^{T}\right)^{-1} \\
& =-\mathbf{A}^{-1} \mathbf{B} \mathbf{Q}^{-1}\left(\mathbf{A}^{-1} \mathbf{B} \mathbf{Q}^{-1}\right)^{T}
\end{aligned}
$$

It is implied in (B.7) that $\mathbf{e}_{i}^{T} \mathbf{A}^{-1} \mathbf{B} \mathbf{A}^{-1} \mathbf{B} \mathbf{A}^{-1} \mathbf{e}_{i}$ is a non-positive real number, yielding

$$
\begin{aligned}
\mathbf{e}_{i}^{T}\left(\mathbf{H}^{H} \mathbf{H}\right)^{-1} \mathbf{e}_{i} & =\mathbf{e}_{i}^{T} \mathbf{A}^{-1} \mathbf{e}_{i}-\mathbf{e}_{i}^{T} \mathbf{A}^{-1} \mathbf{B} \mathbf{A}^{-1} \mathbf{B} \mathbf{A}^{-1} \mathbf{e}_{i} \\
& \geq \mathbf{e}_{i}^{T}\left(\mathbf{Q}^{T} \mathbf{Q}\right)^{-1} \mathbf{e}_{i} .
\end{aligned}
$$

The proof is completed.

[1] D. A. Samo, M. Slimani, G. Baruffa, L. Rugini, A performance study of DVB-T2 and DVB-T2-Lite for mobile reception, Digital Signal Process. 37 (2015) 35-42.

[2] N. Al-Dhahir, H. Minn, S. Satish, Optimum DCT-based multicarrier transceivers for frequency-selective channels, IEEE Trans. Commun. 54 (5) (2006) 911-921.

[3] D. Falconer, S. L. Ariyavisitakul, A. Benyamin-Seeyar, B. Eidson, Frequency domain equalization for single-carrier broadband wireless systems, IEEE Commun. Mag. 40 (4) (2002) 58-66.

[4] X. Ouyang, J. Zhao, Single-tap equalization for fast OFDM signals under generic linear channels, IEEE Commun. Lett. 18 (8) (2014) 1319-1322. 
[5] J. H. Liu, D. K. Yang, Q. Chang, Q. S. Zhang, Research on inter-carrier interference cancellation based on differential coding in OFDM system, Journal of Elec. and Info. Tech. 29 (7) (2007) 1529-1532.

[6] J. Ma, P. V. Orlik, J. Zhang, G. Y. Li, Reduced-rate OFDM transmission for inter-subchannel interference self-cancellation over high-mobility fading channels, IEEE Trans. Wireless Commun. 11 (6) (2012) 2013-2023.

[7] X. Wang, Z. H. Tan, X. Chen, Doppler diversity receiver for broadband wireless OFDM system under high-speed mobile environments, in: Int. Conf. Microwave, Antenna, Propagation and EMC Tech. for Wireless Commun., Beijing, China, 2005, pp. 1444-1447.

[8] W. G. Jeon, K. H. Chang, Y. S. Cho, An equalization technique for orthogonal frequency-division multiplexing systems in time-variant multipath channels, IEEE Trans. Commun. 47 (1) (1999) 27-32.

[9] L. Rugini, P. Banelli, G. Leus, Simple equalization of time-varying channels for OFDM, IEEE Commun. lett. 9 (7) (2005) 619-621.

[10] L. Rugini, P. Banelli, G. Leus, Low-complexity banded equalizers for OFDM systems in Doppler spread channels, EURASIP J. Appl. Signal Process. 2006 (067404) (2006) 1-13.

[11] L. Rugini, P. Banelli, G. Leus, OFDM communications over time-varying channels, Chapter 7 of Wireless Communications over Rapidly TimeVarying Channels, eds, F. Hlawatsch and G. Matz, Academic Press, 2011.

[12] B. Picinbono, P. Chevalier, Widely linear estimation with complex data, IEEE Trans. on Signal Process. 43 (8) (1995) 2030-2033.

[13] F. Sterle, Widely linear MMSE transceivers for MIMO channels, IEEE Trans. on Signal Process. 55 (8) (2007) 4258-4270.

[14] S. Abdallah, I. N. Psaromiligkos, Widely linear versus conventional subspace-based estimation of SIMO flat-fading channels: Mean squared error analysis, IEEE Trans. on Signal Process. 60 (3) (2012) 1307-1318. 
[15] Y. Shi, L. Huang, C. Qian, H. So, Shrinkage linear and widely-linear complex-valued least mean squares algorithms for adaptive beamforming, IEEE Trans. on Signal Process. 61 (1) (2015) 119-131.

[16] Y. S. Choi, P. J. Voltz, F. A. Cassara, On channel estimation and detection for multicarrier signals in fast and selective Rayleigh fading channels, IEEE Trans. Commun. 49 (8) (2001) 1375-1387.

[17] M. Huang, B. B. Li, Block-wise equaliser in fast fading channels, IET Commun. 9 (1) (2015) 108-116.

[18] P. Schniter, Low-complexity equalization of OFDM in doubly selective channels, IEEE Trans. Signal Process. 52 (4) (2004) 1002-1011.

[19] K. Fang, L. Rugini, G. Leus, Low-complexity block turbo equalization for OFDM systems in time-varying channels, IEEE Trans. Signal Process. 56 (11) (2008) 5555-5566.

[20] L. Rugini, P. Banelli, K. Fang, G. Leus, Enhanced turbo MMSE equalization for MIMO-OFDM over rapidly time-varying frequency-selective channels, in: IEEE Int. Workshop on Signal Process. Advances in Wireless Commun. (SPAWC 2009), Perugia, Italy, 2009, pp. 21-24.

[21] H. Doğan, E. Panayirci, H. V. Poor, Low-complexity joint data detection and channel equalisation for highly mobile orthogonal frequency division multiplexing systems, IET Commun. 4 (8) (2010) 1000-1011.

[22] C. Y. Ma, S. W. Liu, C. C. Huang, Low-complexity ICI suppression methods utilizing cyclic prefix for OFDM systems in high-mobility fading channels, IEEE Trans. Veh. Technol. 63 (2) (2014) 718-730.

[23] A. Septimus, Y. Keller, I. Bergel, A spectral approach to inter-carrier interference mitigation in OFDM systems, IEEE Trans. Commun. 62 (8) (2014) 2802-2811. 
[24] J. Z. Zhou, J. Y. Qin, Y. C. Wu, Variational inference-based joint interference mitigation and OFDM equalization under high mobility, IEEE Signal Process. Lett. 22 (11) (2015) 1970-1974.

[25] IEEE Standard for Information Technology-Telecommunications and Information Exchange Between Systems-Local and Metropolitan Area Networks-Specific Requirements Part 11:Wireless LAN Medium Access Control (MAC) and Physical Layer (PHY) Specifications, IEEE 802.11, IEEE 802.11 Working Group (2007).

[26] Frame Structure, Channel Coding and Modulation for a Second Generation Digital Terrestrial Television Broadcasting System (DVB-T2), ETSI Standard, EN 302 755, V1.1.1 (Sep. 2009).

[27] M. Huang, B. B. Li, Research on channel estimation and OFDM signals detection in rapidly time-variant channels, Radioengineering 23 (3) (2014) 880-889.

[28] Z. Tang, R. C. Cannizaro, G. Leus, P. Banelli, Pilot-assisted time-varying channel estimation for OFDM systems, IEEE Trans. Signal Process. 55 (5) (2007) 2226-2238.

[29] Z. Zhang, B. Rao, Sparse signal recovery with temporally correlated source vectors using sparse Bayesian learning, IEEE J. Sel. Topics Signal Process. 5 (5) (2011) 912-926.

[30] X. Zhang, B. Ottersten, Performance analysis of V-BLAST structure with channel estimation errors, in: IEEE Signal Process. Workshop Signal Process. Adv. in Wireless Commun., Rome, Italy, 2003, pp. 487-491.

${ }_{435}$ [31] K. N. Le, K. P. Dabke, BER of OFDM with diversity and pulse shaping in Rayleigh fading environments, Digital Signal Process. 20 (6) (2010) 16871696.

[32] J. Benesty, Y. Huang, J. D. Chen, A fast recursive algorithm for optimum 
sequential signal detection in a BLAST system, IEEE Trans. Signal Process. 51 (7) (2003) 1722-1730.

[33] R. C. Singleton, An algorithm for computing the mixed radix fast Fourier transform, Proc. IEEE 17 (2) (1969) 93-103.

[34] Y. R. Zheng, C. Xiao, Simulation models with correct statistical properties for Rayleigh fading channels, IEEE Trans. Commun. 51 (6) (2003) 920-928.

[35] J. Blogh, L. Hanzo, Third-Generation Systems and Intelligent Wireless Networking: Smart Antennas and Adaptive Modulation, New York: WileyIEEE Press, 2002.

[36] X. Cai, G. B. Giannakis, Bounding performance and suppressing intercarrier interference in wireless mobile OFDM, IEEE Trans. Commun. 51 (12) (2003) 2047-2056.

[37] R. Logeshwaran, I. J. L. Paul, Performance study on the suitability of Reed Solomon Codes in WiMAX, in: Int. Conf. on Wireless Commun. and Sensor Computing, Chennai, India, 2010, pp. 1-4.

[38] E. Eraslan, B. Daneshrad, C. Y. Lou, Performance indicator for MIMO MMSE receivers in the presence of channel estimation error, IEEE Wireless Commun. lett. 2 (2) (2013) 211-214.

[39] C. Wang, E. K. S. Au, R. D. Murch, W. H. Mow, R. S. Cheng, V. Lau, On the performance of the MIMO zero-forcing receiver in the presence of channel estimation error, IEEE Trans. Wireless Commun. 6 (3) (2007) 805-810. 